\title{
Molecular Phylogeny and Phenotypic Characterization of Yeasts with a Broad Range of pH Tolerance Isolated from Natural Aquatic Environments
}

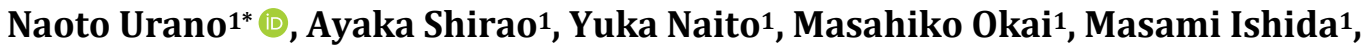 \\ Masachika Takashio
}

\author{
${ }^{1}$ Department of Ocean Sciences, Laboratory of Marine Biochemistry, Tokyo University of Marine Science and Technology, \\ Tokyo, Japan \\ ${ }^{2}$ Zensho Laboratories of Food Technology, Zensho Holdings Co. Ltd., Tokyo, Japan \\ Email: *urano@kaiyodai.ac.jp
}

How to cite this paper: Urano, N., Shirao, A., Naito, Y., Okai, M., Ishida, M. and Takashio, M. (2019) Molecular Phylogeny and Phenotypic Characterization of Yeasts with a Broad Range of $\mathrm{pH}$ Tolerance Isolated from Natural Aquatic Environments. Advances in Microbiology, 9, 56-73. https://doi.org/10.4236/aim.2019.91005

Received: December 19, 2018

Accepted: January 13, 2019

Published: January 16, 2019

Copyright $\odot 2019$ by author(s) and Scientific Research Publishing Inc. This work is licensed under the Creative Commons Attribution International License (CC BY 4.0).

http://creativecommons.org/licenses/by/4.0/

\begin{abstract}
In this study, yeasts with broad range of $\mathrm{pH}$ tolerance were isolated and characterized from natural aquatic environments in Japan. Only a few basic and application studies of alkali-tolerant yeasts have been reported, despite the unmet industrial needs. First, we surveyed alkali-tolerant yeasts from natural aquatic environments at $\mathrm{pH} 7.6$ - 9.4. We isolated 35 yeast strains that grew in pH 9.0 medium, from seven genera and nine species: 25 strains (N1, N2, through N6, N9, K1, K3 through K19) were Rhodotorula mucilaginosa; one (N7) was Rhodosporidium fluvial; one (N8) was Scheffersomyces spartinae; two (N10 and N13) were Wicherhamonyces anomalus, one (N11) was Cyberlindnera saturnus, one (S1) was Candida sp.; two (S2 and S4) were Candida intermedia; one (S3) was Candida quercuum; and one (K2) was Cryptococcus liquefacience. We examined the effects of $\mathrm{pH}$ on the growth of representative yeast strains. Strains K12 and S4 showed high growth at $\mathrm{pH} 3-10$. Strains N7, $\mathrm{N} 8, \mathrm{~N} 10, \mathrm{~N} 11$, and S3 showed high growth at $\mathrm{pH} 3-9$. Strains K2 and S1 showed high growth at $\mathrm{pH} 4-8$. All nine of these strains had neutralizing activities from acidic media at $\mathrm{pH} 3-5$ to $\mathrm{pH} 6-8$. We previously isolated acid-tolerant yeasts (Cryptococcus sp. T1 [1] and Candida intermedia CeA16 [2]) from extremely acidified environments; they showed high growth at $\mathrm{pH} 3$ - 9 and neutralizing activities of acidic media by releasing ammonium ions. Thus, alkali-tolerant yeasts and acid-tolerant yeasts were found to be similar species and have both high growth at a broad range of $\mathrm{pH}$ and neutralizing activities of acid media. Previously, we also isolated acid-tolerant, acid-neutralizing yeasts
\end{abstract}


from neutral natural environments (26 strains, 12 species) [3]. Next, we constructed the phylogenetic trees of both acid-tolerant yeasts and alkali-tolerant yeasts. All were situated in the same classification position. Similar yeast species with a broad range of $\mathrm{pH}$ tolerance were living in natural aquatic environments at $\mathrm{pH}$ values from alkali to acid.

\section{Keywords}

pH Tolerance, Yeast Species, Aquatic Environment, Phylogenetic Tree, Fermentation

\section{Introduction}

Various types of yeasts and yeast-like microorganisms are widely spread in nature, and some have been used since $2000 \mathrm{BC}$ for fermented products (e.g. alcoholic beverages and the leavening of bread). Over the past several decades, suitable yeasts for different food processes have been repeatedly isolated and bred separately according to the type of fermentation desired. These efforts revealed that all industrial-use yeasts should have similar characteristics of high fermentative activity and high tolerance activities under various types of stress. Identification methods for yeasts were developed after the 18th century, and most of the yeasts used in the fermentation industries were found to be a species of Saccharomyces cerevisiae. From ancient to modern times, $S$. cerevisiae has been the most important microorganism species in the history of humans.

However, with the progress of the bioethanol industry in recent years, the breeding of novel yeast strains other than $S$. cerevisiae with higher fermentative activity under several stress pressures is needed, because various types of wasted biomass materials are used as fermentation substrates, and from an economical point of view the concentration of the biomass should be higher. Above all, in bioethanol production, acids or alkalis are used for the hydrolysis of a cellulosic biomass, and the direct fermentation of hydrolysates using yeasts without neutralizing themselves is desirable. Fermentative yeasts with $\mathrm{pH}$ tolerance are thus considered potential beneficial candidates for efficient bioethanol production. Several studies of acid-tolerant yeasts have been conducted, as $S$. cerevisiae and other high-fermentative yeasts show high activity in neutral or acid environments ( $\mathrm{pH} 4$ - 7) [4] [5] [6]. Only a few basic and application studies of alkali-tolerant yeasts have been reported, despite the unmet industrial needs [7].

In previous investigations, we isolated and characterized two strains of acid-tolerant yeasts from extremely acidic environments. One strain was Cryptococcus sp. T1 from Lake Tazawa in Japan's Akita prefecture, is a caldera lake polluted with hydrochloric acid from an upstream hot spring [1]. The other yeast strain is Candida fluviatilis CeA16 from Agatsuma River in Japan's Gunma prefecture, which is polluted with sulfuric acid from Mount Kusatsu-Shirane [2]. Both strains T1 and CeA16 showed high growth at $\mathrm{pH} 3-9$, 
and both showed neutralizing activity from acidic media to $\mathrm{pH} 6$ - 8 by releasing ammonium ions.

We also previously isolated and characterized 26 strains and 12 species of acid-tolerant yeasts that had neutralizing activities of acidic media from neutral environments in the city of Yokohama, Japan [3]. The acid-tolerant and acid-neutralizing yeasts were found to exist in both acidic and neutral environments.

In the present study, we attempted to isolate alkali-tolerant yeasts from alkali environments ( $\mathrm{pH} 7.6$ - 9.4) in Japan's Kanto region. We characterized the yeast strains' growth and fermentation activities, and we constructed the strains' phylogenetic trees and compared them with those of acid-tolerant yeasts.

\section{Materials and Methods}

\subsection{Collection of Environmental Samples}

We surveyed alkali aquatic environments near metropolitan areas and selected four stations in Japan in order to isolate alkali-tolerant yeasts. In May 2016, we collected water and sediment samples from four aquatic environments in Japan's Kanto region: Station \#1 (a pond on the campus of Tokyo University of Marine Science and Technology, Tokyo), Station \#2 (at the coast of Kasairinkai Park, Tokyo), Station \#3 (at the coast of Senbongi Park in Shizuoka Prefecture, Japan), and Station \#4 (at the coast of the Shioiri River in Tateyama of Chiba Prefecture, Japan). All samples were collected in sterile plastic tubes. We measured the samples' temperature, $\mathrm{pH}$ values, and $\mathrm{NaCl}$ concentration. The samples were immediately transferred to and stored at Tokyo University of Marine Science and Technology at $<4^{\circ} \mathrm{C}$.

\subsection{Medium Culture}

For the cultivation of yeasts in the water and sediment samples, we used a YPD medium consisting of $1.0 \% \mathrm{w} / \mathrm{v}$ yeast extract, $2.0 \% \mathrm{w} / \mathrm{v}$ proteose peptone (Becton Dickinson, Lincoln Park, NJ, USA), and 2.0\% w/v D-(+)-glucose (Kokusan Chemicals, Tokyo). For the isolation of yeasts from the environments, $0.01 \% \mathrm{w} / \mathrm{v}$ chloramphenicol (Wako Pure Chemical Industries, Tokyo) was added to the YPD medium to prevent the growth of bacteria. The $\mathrm{pH}$ of the media was adjusted with sulfuric acid or sodium hydroxide (Wako). Solid media were constructed by adding $2.0 \% \mathrm{w} / \mathrm{v}$ agar (Kanto Chemicals, Tokyo) to the YPD liquid medium.

\subsection{Isolation of Alkali-Tolerant Yeast Strains}

The water samples were filtered through a $0.45-\mu \mathrm{m}$ FTFE membrane-filter (Advantec, Tokyo), and microorganisms were trapped on the filter. The microorganisms were dispersed into the portion of the filtrate by a mixer; thus, we obtained an approx. 100-fold-concentrated population of the microorganisms in the water samples. To obtain a moderate population of the microorganisms, we 
diluted sediment samples to 10 -fold with physiological saline $(0.8 \% \mathrm{w} / \mathrm{v} \mathrm{NaCl})$.

For the first screening of alkali-tolerant yeasts, a $200-\mu \mathrm{l}$ volume of each preparation was spread on the YPD solid medium containing chloramphenicol at $\mathrm{pH}$ 8.0 and incubated at $25^{\circ} \mathrm{C}$. After several days' cultivation, growing yeast-like colonies were picked up, and we observed their cells under a light microscope. Cells that were yeast-like morphologically were isolated and stored at $-80^{\circ} \mathrm{C}$.

For the second screening of alkali-tolerant yeasts, the isolates obtained by the first screening were inoculated on the YPD solid medium at $\mathrm{pH} 9.0$ and incubated at $25^{\circ} \mathrm{C}$. The growing colonies were obtained as candidate alkali-tolerant yeasts, stored at $-80^{\circ} \mathrm{C}$, and numbered as members in the yeast collection.

\subsection{Yeast Identification}

The $28 \mathrm{~S}$ rRNA genes of the isolates in the yeast library were amplified by polymerase chain reactions (PCRs) using the forward primer NL-1 (5'-GCATATCAATAAGCGGAGGAAAAG-3') and the reverse primer NL-4 (5'-GGTCCGTGTTTCAAGACGG-3') and Premix Ex Taq (Takara Bio, Shiga, Japan). The $28 \mathrm{~S}$ rRNA phylogenetic tree was constructed in the molecular evolutionary genetics analysis (MEGA) tool 6.06 using the maximum likelihood method with a 1000 replicate bootstrap resampling. The D1/D2 domain sequences of the $28 \mathrm{~S}$ rRNA genes in the yeasts were deposited in DDBJ, EMBL, and GenBank.

\subsection{Fermentation Activities of the Yeasts}

Each isolate in the yeast library was inoculated into $10 \mathrm{ml}$ of YPD liquid medium with a Durham pipe in a test tube and then anaerobically incubated at $25^{\circ} \mathrm{C}$. After 7 days' cultivation, the yeast fermentative activity in the medium was examined by the naked eye based on the storage of gas $\left(\mathrm{CO}_{2}\right)$ evolving from the cells into the Durham pipe.

\subsection{Yeast Growth Tests}

Each strain in the yeast library was precultured in YPD medium at $25^{\circ} \mathrm{C}$ for $24 \mathrm{hr}$. The growing cells were precipitated by centrifugation at $3000 \mathrm{rpm}$ for 5 min and then washed with physiological saline. The centrifugation/washing procedure was conducted three times, and the cell precipitates were obtained. A $100-\mu \mathrm{g}$ portion of the wet cells was inoculated into $10 \mathrm{ml}$ of YPD liquid medium with the $\mathrm{pH}$ value $3.0,4.0,5.0,6.0,7.0,8.0,9.0$, or 10.0. We measured the growth curves of the yeasts at $25^{\circ} \mathrm{C}$ for $72 \mathrm{hr}$ using a bio-photorecorder (temperature-gradient incubator, Advantec, Japan).

\subsection{Measurement of $\mathrm{pH}$ in the Yeast Cultures}

In the growth tests of the yeasts in the YPD liquid media with the initial $\mathrm{pH}$ values 3.0, 4.0, 5.0, 6.0, 7.0, 8.0, 9.0, and 10.0, we measured the change of $\mathrm{pH}$ values in the media after 3 days' cultivation at $25^{\circ} \mathrm{C}$ by using a LAQUA pH meter (F-71, Horiba Scientific, Fukuoka, Japan). 


\section{Results}

\subsection{Isolation and Identification of Alkali-Tolerant Yeasts}

Table 1 shows the $\mathrm{pH}$, temperature, and $\mathrm{NaCl}$ concentration of the sampling areas and the isolation of alkali-tolerant yeast-like microorganisms. The first screening by the isolation of growing colonies on the YPD solid medium containing chloramphenicol at $\mathrm{pH} 8.0$ identified 22, 229, 37, and 21 yeast-like isolates from both water and sediment samples taken from Stations \#1, \#2, \#3, and \#4, respectively. In the second screening at $\mathrm{pH} 9.0$ of the isolates identified by the first screening of growing yeast-like cells, 19, 12, and four strains were obtained from Stations \#2, \#3, and \#4, respectively. Our microscopy observations of the cell morphology suggested that all 35 of the second-screening strains were yeast species with alkali tolerance. We numbered the strains as K1-K19 from Station \#2, as N1-N11 and N13 from Station \#3, and as S1-S4 from Station \#4.

No alkali-tolerant yeasts were isolated from Station \#1, even though the $\mathrm{pH}$ value of the water at that station was 9.4. At that station, we observed highly concentrated microalgae growing in the pond, the $\mathrm{pH}$ of which was 9.4 due to ammonium ions being released from the microalgae, and few alkali-tolerant yeasts seemed to be living at this station.

Table 2 shows the identification results for the 35 strains of alkali-tolerant yeast. These strains were identified as members of seven genera and nine species: 25 strains (N1, N2, N3, N4, N5, N6, N9, K1, K3, K4, K5, K6, K7, K8, K9, K10, K11, K12, K13, K14, K15, K16, K17, K18, and K19) were Rhodotorula mucilaginosa. One strain (N7) was Rhodosporidium fluviale. One strain (N8) was Scheffersomyces spartinae. Two strains (N10 and N13) were Wicherhamonyces anomalus. One strain N11 was Cyberlindnera saturnus. One strain (S1) was Candida sp. Two strains (S2 and S4) were Candida intermedia. One strain (S3) was Candida quercuum. One strain (K2) was Cryptococcus liquefacience. Therefore, Rhodotorula mucilaginosa seemed to be a common species among alkali-tolerant yeasts.

\subsection{The Fermentation Activities of the Alkali-Tolerant Yeasts}

Table 3 summarizes the fermentation activities of the 35 strains of alkali-tolerant

Table 1. $\mathrm{pH}$, temperature, and $\mathrm{NaCl}$ concentration of sampling area and isolation of alkali-tolerant yeasts.

\begin{tabular}{ccccccc}
\hline $\begin{array}{c}\text { Sampling } \\
\text { area }\end{array}$ & $\mathrm{pH}$ & $\begin{array}{c}\mathrm{NaCl} \\
\text { concentration } \\
\mathbf{( \% )}\end{array}$ & $\begin{array}{c}\text { Temp. } \\
\cdot \mathrm{C}\end{array}$ & $\begin{array}{c}\text { No. of isolates } \\
\text { at } \mathrm{pH} \mathrm{8.0}\end{array}$ & $\begin{array}{c}\text { No. of } \\
\text { isolates at } \\
\mathrm{pH} \mathrm{9.0}\end{array}$ & Strain \\
\hline Station \#1 & 9.40 & 0.01 & 26.0 & 22 & 0 & - \\
Station \#2 & 7.65 & 1.40 & 22.4 & 229 & 19 & K1-K19 \\
Station \#3 & 7.55 & 2.70 & 20.3 & 37 & 12 & N1-N11, N13 \\
Station \#4 & 8.08 & 0.25 & ND & 21 & 4 & S1-S4 \\
\hline
\end{tabular}

Station \#1: A pond in Tokyo university of marine science and Technplogy in Tokyo, Japan; Station \#2: A coast of the Kasairinkai Park in Tokyo, Japan; Station \#3: The coast at Senbongi Park in Shizuoka Prefecture, Japan; Station \#4: The coast of the Shioiri River in Tateyama of Chiba Prefecture, Japan. 
Table 2. Identification results for 35 strains of alkali-tolerant yeasts.

\begin{tabular}{|c|c|c|c|}
\hline Strain & Identification result & Identity (\%) & Accession No. \\
\hline N1 & & & LC438608 \\
\hline $\mathrm{N} 2$ & & & LC438609 \\
\hline N3 & & & LC438610 \\
\hline N4 & & & LC438611 \\
\hline N5 & & & LC438612 \\
\hline N6 & & & LC438613 \\
\hline N9 & & & LC438614 \\
\hline K1 & & & LC438615 \\
\hline K3 & & & LC438617 \\
\hline K4 & & & LC438618 \\
\hline K5 & & & LC438619 \\
\hline K6 & & & LC438620 \\
\hline K7 & Rhodotorula mucilaginosa & $99-100$ & LC438621 \\
\hline K8 & & & LC438622 \\
\hline K9 & & & LC438623 \\
\hline K10 & & & LC438624 \\
\hline K11 & & & LC438625 \\
\hline K12 & & & LC438626 \\
\hline K13 & & & LC438627 \\
\hline K14 & & & LC438628 \\
\hline K15 & & & LC438629 \\
\hline K16 & & & LC438630 \\
\hline K17 & & & LC438631 \\
\hline K18 & & & LC438632 \\
\hline K19 & & & LC438633 \\
\hline N7 & Rodosporidium fluviale & 100 & LC438634 \\
\hline N8 & Scheffersomyces spartinae & 100 & LC438635 \\
\hline N10 & WTickerhamomuces anomalus & 100 & LC438636 \\
\hline N13 & 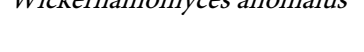 & 100 & LC438637 \\
\hline N11 & Cyberlindnera saturnus & 100 & LC438638 \\
\hline S1 & Candida sp. & 100 & LC438639 \\
\hline S2 & & 100 & LC438640 \\
\hline S4 & Candida intermedia & 100 & LC438641 \\
\hline S3 & Candida quercuum & 99 & LC438642 \\
\hline $\mathrm{K} 2$ & Cryptococcus liquefaciens & 100 & LC438616 \\
\hline
\end{tabular}


Table 3. Fermentation activities of 35 strains of alkali-tolerant yeasts.

\begin{tabular}{|c|c|c|c|}
\hline Sampling area & Species & Strain & Fermentation \\
\hline & Rhodotorula mucilaginosa & K1 & - \\
\hline & Cryptococcus liquefaciens & K2 & - \\
\hline & & K3 & - \\
\hline & & $\mathrm{K} 4$ & - \\
\hline & & K5 & - \\
\hline & & K6 & - \\
\hline & & K7 & - \\
\hline & & K8 & - \\
\hline & & K9 & - \\
\hline \multirow[t]{16}{*}{ Station \#2 } & & K10 & - \\
\hline & Rhodotorula mucilaginosa & K11 & - \\
\hline & & K12 & - \\
\hline & & K13 & - \\
\hline & & K14 & - \\
\hline & & K15 & - \\
\hline & & K16 & - \\
\hline & & K17 & - \\
\hline & & K18 & - \\
\hline & & K19 & - \\
\hline & & N1 & - \\
\hline & & $\mathrm{N} 2$ & - \\
\hline & Rhodotorula mucilaginos & N3 & - \\
\hline & НН & N4 & - \\
\hline & & N5 & - \\
\hline & & N6 & - \\
\hline \multirow[t]{8}{*}{ Station \#3 } & Rodosporidium fluviale & N7 & - \\
\hline & Scheffersomyces spartinae & $\mathrm{N} 8$ & + \\
\hline & Rhodotorula mucilaginosa & N9 & - \\
\hline & Wickerhamomyces anomalus & N10 & + \\
\hline & Cyberlindnera saturnus & N11 & + \\
\hline & Wickerhamomyces anomalus & N13 & + \\
\hline & Candidasp. & S1 & + \\
\hline & Candida intermedia & S2 & + \\
\hline \multirow[t]{2}{*}{ Station \#4 } & Candida quercuum & S3 & + \\
\hline & Candida intermedia & S4 & + \\
\hline
\end{tabular}

(+): with fermentation activity; (-): without fermentation activity.

yeast. Among them, 27 strains and three species had no fermentative activity, but eight strains and six species had fermentative activities in the media: Schef- 
fersomyces spartinae N8, Wicherhamonyces anomalus N10 and N13, Cyberlindnera saturnus N11, Candida sp. S1, Candida intermedia S2 and S4, and Candida quercuum $\mathrm{S} 3$. We considered these eight yeast strains as candidates for bioethanol production.

\subsection{Effect of $\mathrm{pH}$ in the Media on the Growth of Alkali-Tolerant Yeasts}

Using the representative strains of nine species described above in Section 3.1, we examined the effect of $\mathrm{pH}$ on the growth of the strains. The growth curve of each of the nine strains is provided in Figures 1-9. Two strains (Rhodotorula mucilaginosa K12 and Candida intermedia S4) showed high growth at pH 3 - 10 (Figure 1 and Figure 2). Five strains (Rhodosporidium fluviale N7, Scheffersomyces spartinae N8, Wicherhamonyces anomalus N10, Cyberlindnera saturnus N11, and Candida quercuum S3) showed high growth at pH 3 - 9 (Figures 3-7). Two strains (Cryptococcus liquefacience K2 and Candida sp. S1) showed high growth at pH 4 - 8 (Figure 8 and Figure 9). Thus, all nine species isolated as alkali-tolerant yeasts in this study were found to have both alkali tolerance and acid tolerance.

Most importantly, Rhodotorula mucilaginosa K12 and Candida intermedia S4 had high growth activities at the broad range of $\mathrm{pH}$ (3.0 to 10.0), and strain S4 also had fermentation activities. Saito et al. reported that $C$. intermedia 4-6-4T2, an acid-tolerant mutant from parent strain $C$. intermedia 10601, was obtained from strain 10,601 by repeated fermentation and cultivation under the addition of acetic acid. They observed effective ethanol production by $C$. intermedia 4-6-4T2 from hemicellulose hydrolysate containing both glucose and xylose.

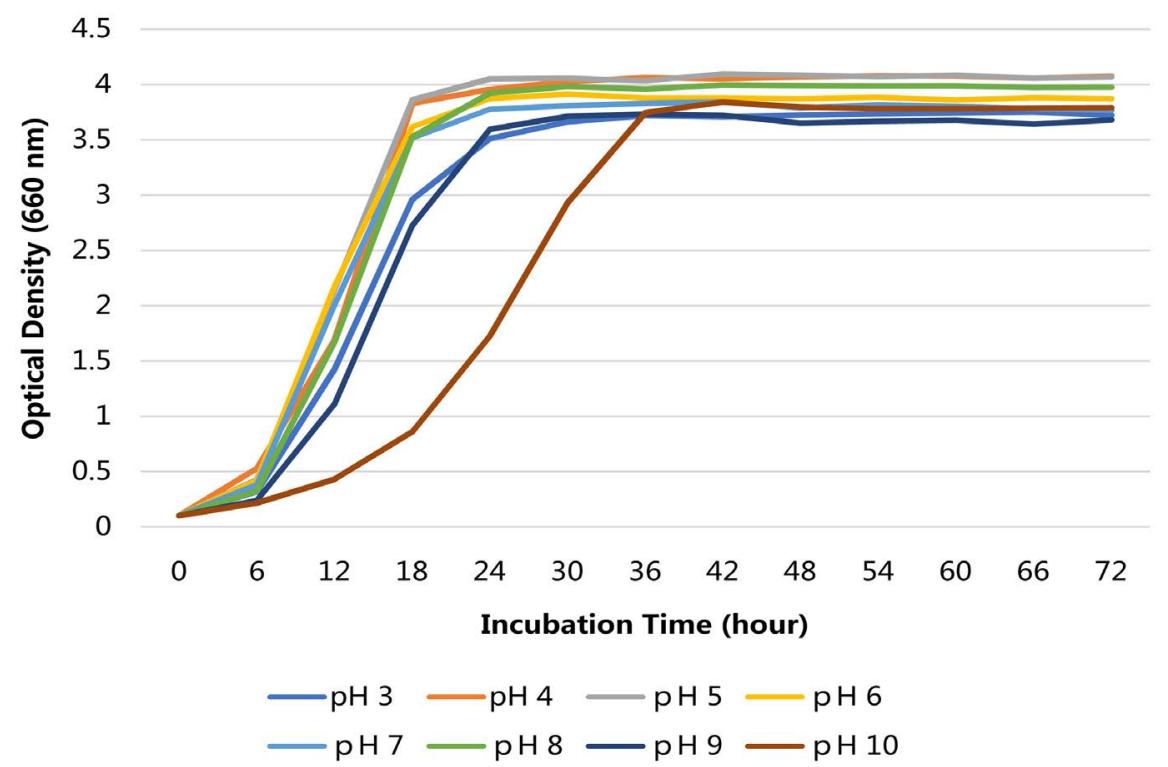

Figure 1. The growth of Rhodotorula mucilaginosa K12 in YPD medium at initial pH values ranging from 3.0 to 10.0 . The values are the mean of triplicate cultures. The standard deviation (SD) values were omitted in order to clarify the growth curves. 


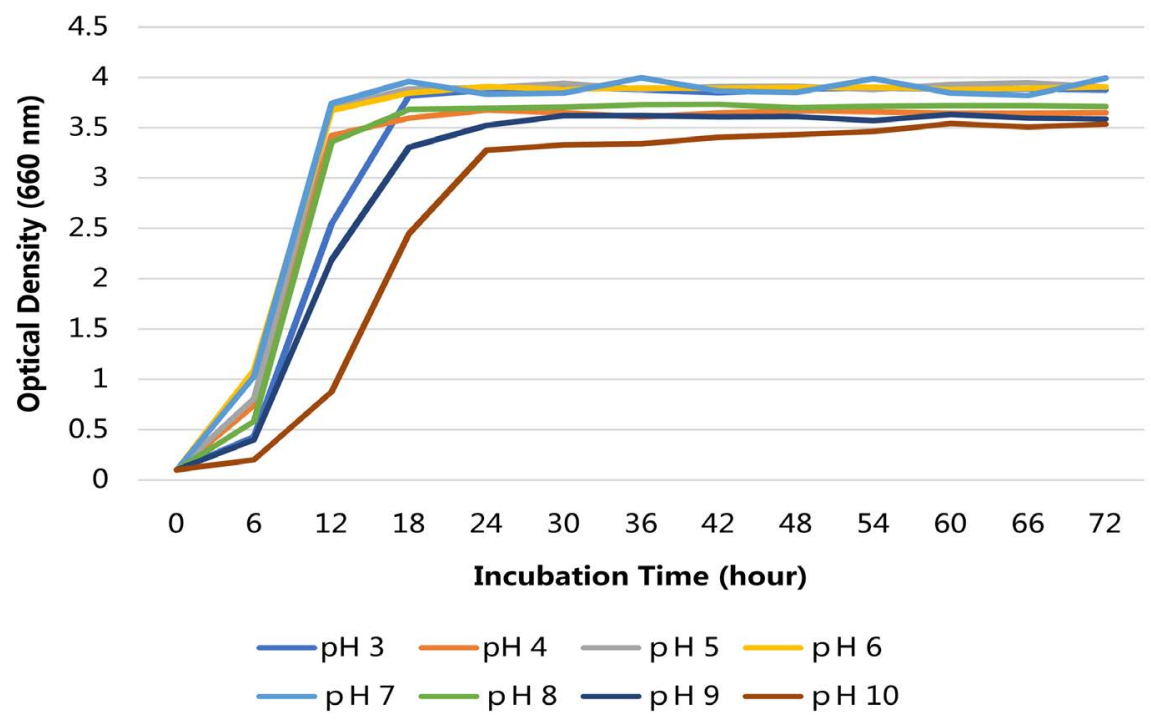

Figure 2. The growth of Candida intermedia S4 in YPD medium at initial pH values ranging from 3.0 to 10.0. The values are the mean of triplicate cultures. The SD values were omitted in order to clarify the growth curves.

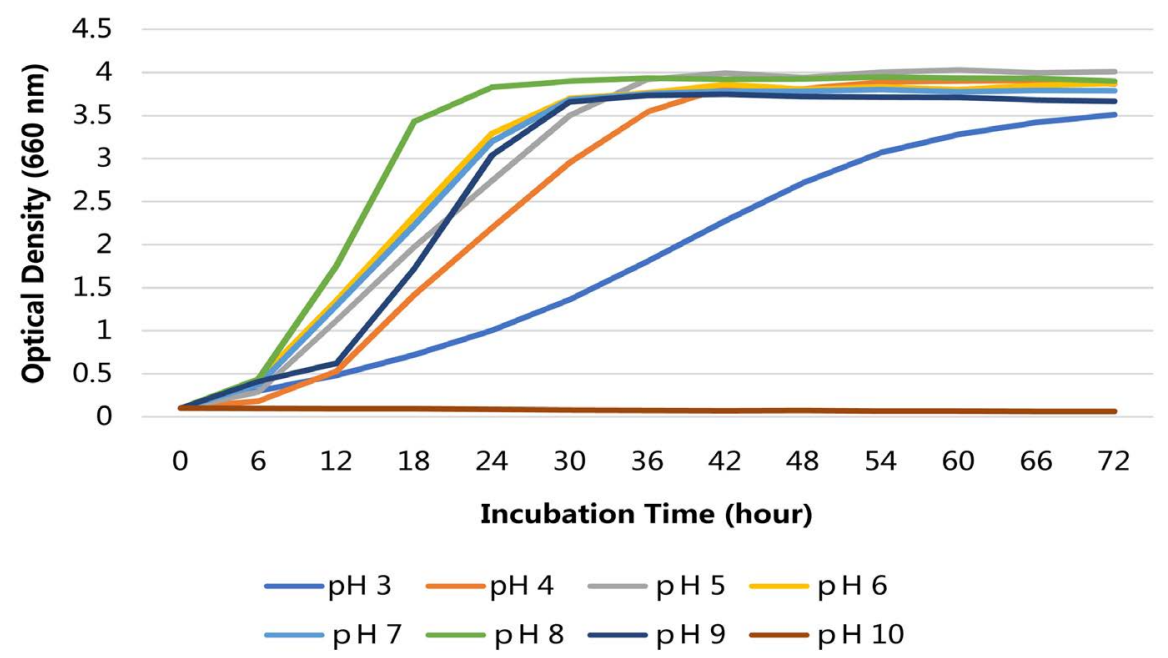

Figure 3. The growth of Rhodosporidium fluviale N7 in YPD medium at initial pH values ranging from 3.0 to 10.0. The values are the mean of triplicate cultures. The SD values were omitted in order to clarify the growth curves

Strain 4-6-4T2 simultaneously assimilated $25 \mathrm{~g} / \mathrm{L}$ glucose and $25 \mathrm{~g} / \mathrm{L}$ xylose and produced ethanol at $1.0 \mathrm{~g} / \mathrm{L} / \mathrm{hr}$, with a total of $50 \mathrm{~g} / \mathrm{L}$ [8]. We thus considered Candida intermedia S4 the most suitable candidate for effective bioethanol from waste cellulosic biomass.

Lisichkina et al. reported alkali-tolerant yeasts from natural biotopes, i.e. soda-rich soils of Armenia and the Transbaikal region of Russia [9]. They cultured the yeast in $\mathrm{pH} 10.0$ medium, and the yeasts were identified as Cryptococcus laurentii, Candida albicans, Rhodotolula glutinis, Rhodotolula mucilaginosa, and Sporobolemyces roseus. We observed that these yeasts' species are in good agreement with those of the alkali-tolerant yeasts in the present study. 


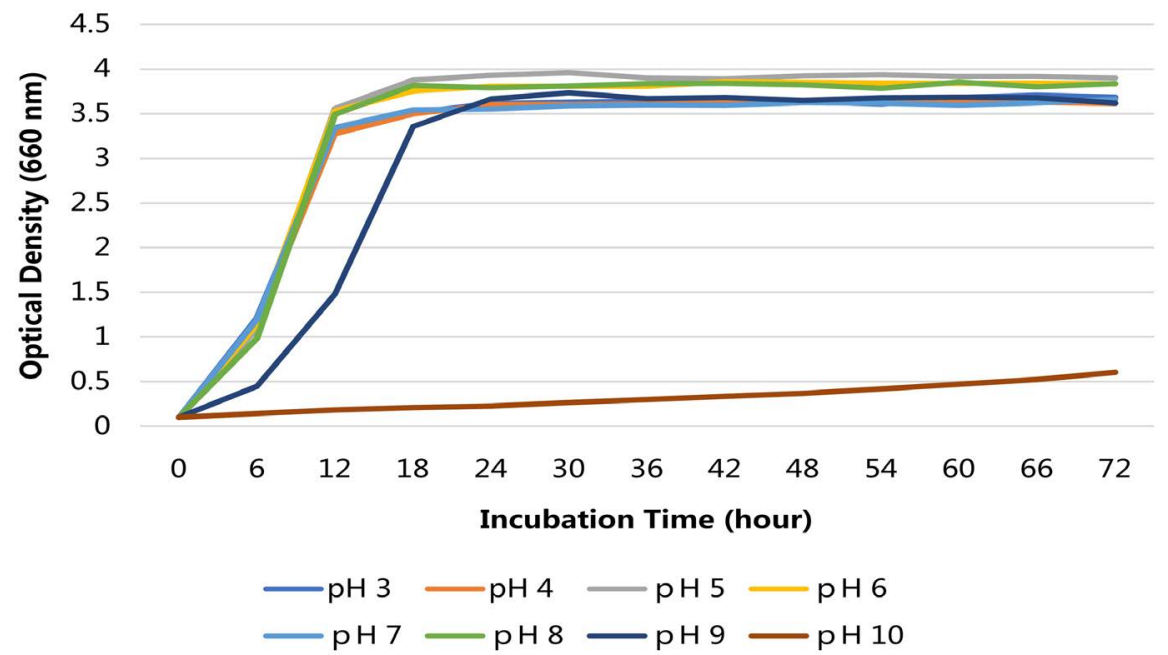

Figure 4. The growth of Scheffersomyces spartinae N8 in YPD medium at initial pH values ranging from 3.0 to 10.0. The values are the mean of triplicate cultures. The SD values were omitted in order to clarify the growth curves.

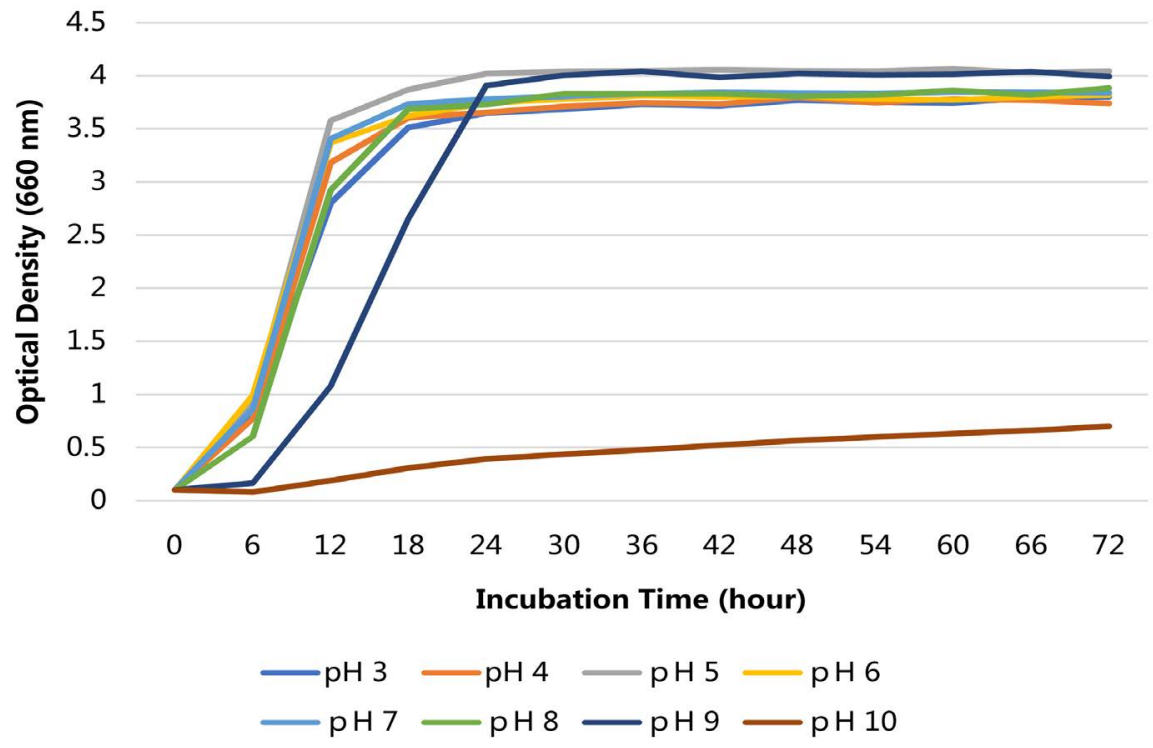

Figure 5. The growth of Wicherhamonyces anomalus N10 in YPD medium at initial pH values ranging from 3.0 to 10.0. The Values are the mean of triplicates (Standard deviation was omitted to clarify the growth curves).

\subsection{Changes in the $\mathrm{pH}$ Values in the Yeast Cultures}

We cultured representative strains of nine species at the initial $\mathrm{pH}$ values of 3.0, $4.0,5.0,6.0,7.0,8.0,9.0$ and 10.0 and then measured the changes of the $\mathrm{pH}$ values after 3 days' cultivation (Table 4). At initial $\mathrm{pH} 3.0$, the $\mathrm{pH}$ of three strains $(\mathrm{K} 2, \mathrm{~K} 12$, and $\mathrm{N} 11)$ increased to $>4.0$ in the media. At the initial $\mathrm{pH} 4.0$, the $\mathrm{pH}$ of two other strains (N7 and N11) increased to $>7.0$ in the media. At the initial $\mathrm{pH} 5.0$, the $\mathrm{pH}$ of eight strains (S1, S4, K2, K12, N7, N8, N10, and N11) increased to $>7.0$ in the media. At the initial $\mathrm{pH} 6.0$, the $\mathrm{pH}$ of all nine strains increased to $>7.0$ in the media. Therefore, all nine strains were observed to have 


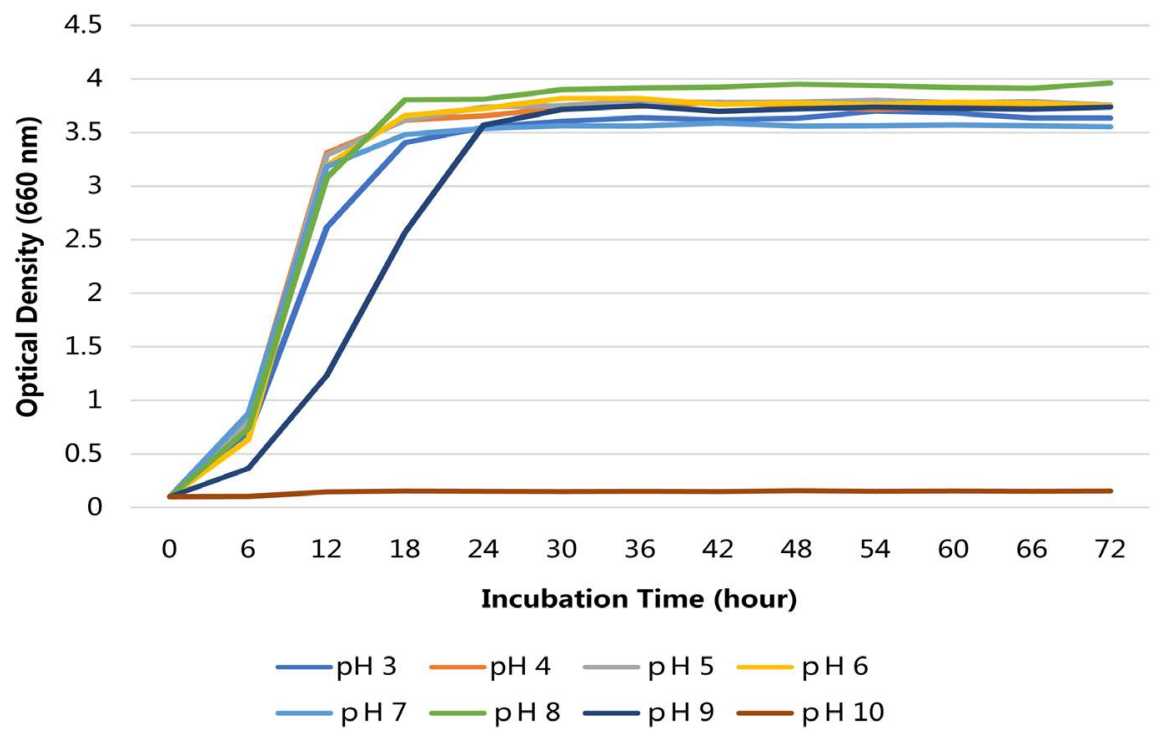

Figure 6. The growth of Cyberlindnera saturnus N11 in YPD medium at initial pH values ranging from 3.0 to 10.0. The values are the mean of triplicate cultures. The SD values were omitted in order to clarify the growth curves.

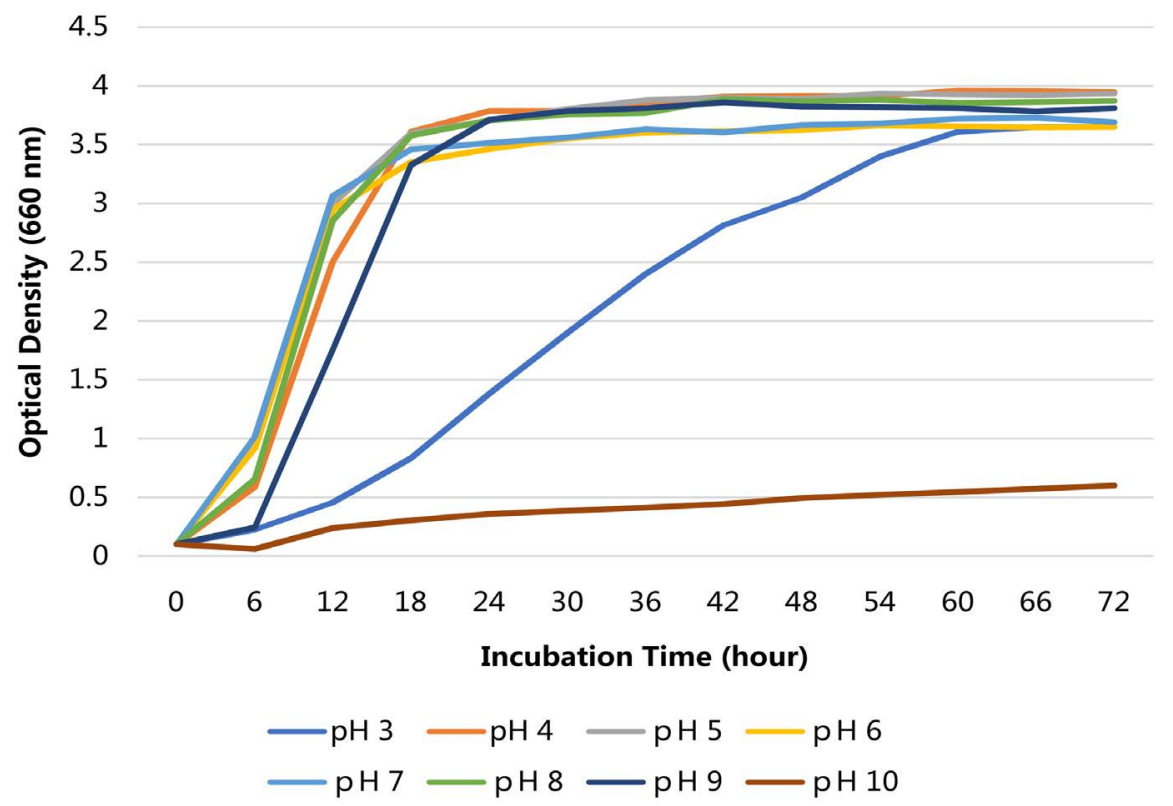

Figure 7. The growth of Candida quercuum S3 in YPD medium at initial pH values ranging from 3.0 to 10.0. The values are the mean of triplicate cultures. The SD values were omitted in order to clarify the growth curves.

acid-neutralizing activities by releasing ammonium ions (data not shown).

\subsection{Polygenetic Tree of $\mathrm{pH}$-Tolerant Yeasts}

The nine species of alkali-tolerant yeasts had $\mathrm{pH}$ tolerance over a broad range of $\mathrm{pH}$, and their genera and species were very similar to those of the acid-tolerant yeasts that were isolated previously [1] [2] [3]. The yeasts that we used for construction of the polygenetic tree are summarized in Table 5. The polygenetic 


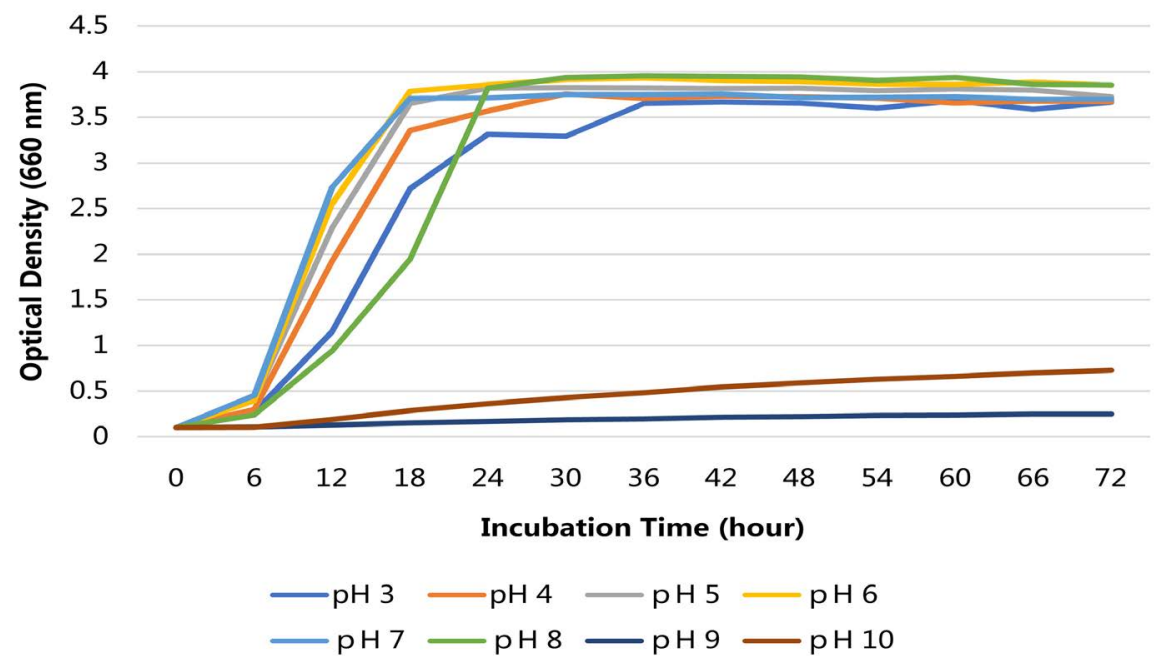

Figure 8. The growth of Cryptococcus liquefacience K2 in YPD medium at initial $\mathrm{pH}$ values ranging from 3.0 to 10.0 . The values are the mean of triplicate cultures. The SD values were omitted in order to clarify the growth curves.

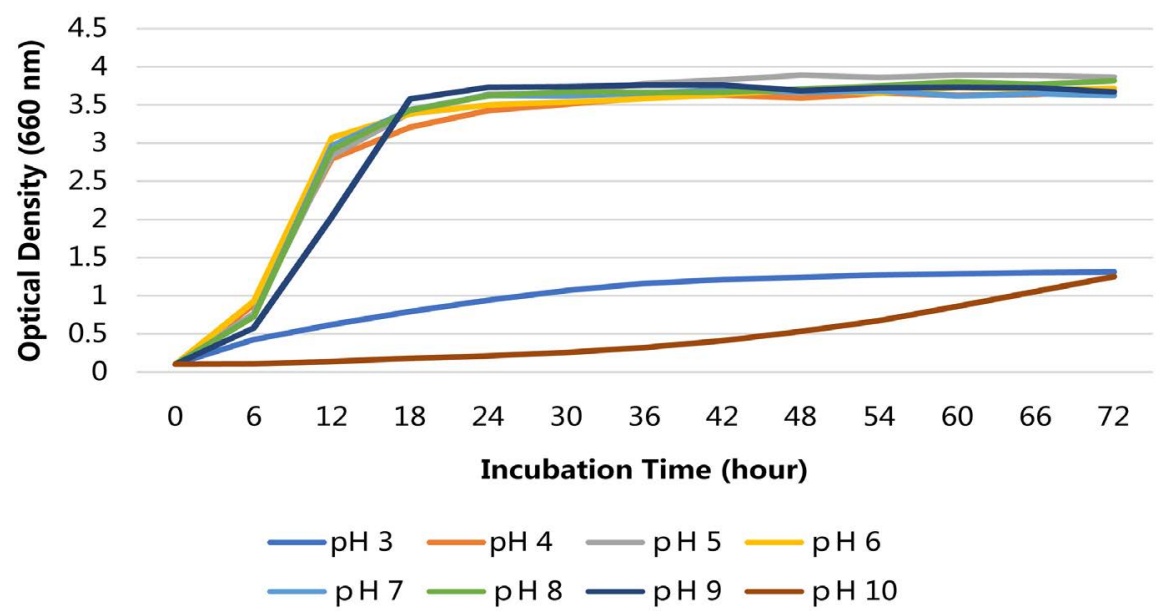

Figure 9. The growth of Candida sp. S1 in YPD medium at initial $\mathrm{pH}$ values ranging from 3.0 to 10.0. The values are the mean of triplicate cultures. The SD values were omitted in order to clarify the growth curves.

trees of the acid-tolerant yeasts (26 strains) and alkali-tolerant yeasts (nine strains) are shown in Figure 10. These trees were constructed on the 28s rRNA gene sequences by the maximum likelihood algorithm in MEGA version 6.06.

Based on these results, both acid-tolerant yeasts and alkali-tolerant yeasts were situated in the same classification position. Of the nine strains, $\mathrm{K} 2$ was the sister group of the clade that includes Filobassidium magnum mi-w16. K12 and N7 were the sister group that includes Rhodotolura sp. n-w29, si-w12, Leucosporidium golubevii si-w13, and Microbotryozyma collariae sm-w40. The strains S1, S3, S4, N8, N10, and N11 were the sister group that includes Candida sp. om-w46, h-m7, Meyerozyma guilliemondii mr-w1, Candida parapsiosis h-m7, and Candida oleophila n-w33. Thus, similar yeast species with a broad range of $\mathrm{pH}$ tolerance were found to be living in natural aquatic environments at a broad 


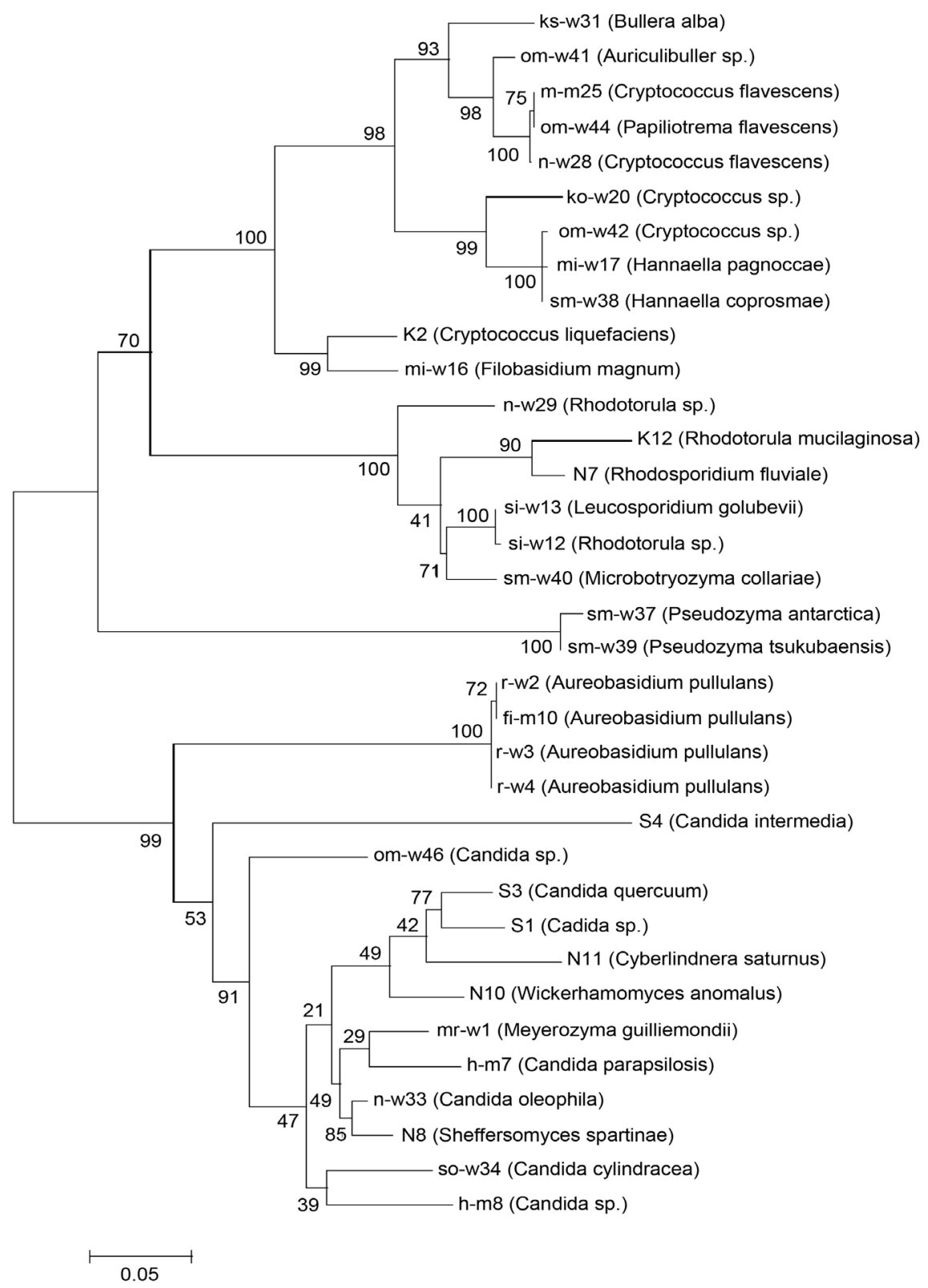

Figure 10. The polygenetic tree of both acid-tolerant yeasts (26 strains) and alkali-tolerant yeasts (nine strains). It was constructed on the $28 \mathrm{~s}$ rRNA gene sequences by the maximum likelihood algorithm in MEGA ver. 6.06.

range of $\mathrm{pH}$ from alkali to acid.

\section{Discussion}

We have been studying the characterization and application of aquatic yeasts that could be suitable for bioethanol production, and we developed several types of bioethanol production systems that use cellulosic biomass such as seaweeds, i.e. Undaria pinnafida [10] [11] [12], Ulva spp., and Costaria costata [10] [11] [12], an alien aquatic plant in Japan, Eichhornia crassipes [13] [14]; and paper or wood scrap [11] [15] along with aquatic yeasts. In bioethanol production from a 
Table 4. Change of $\mathrm{pH}$ after 3 days' cultivation of alkali-tolerant yeasts.

\begin{tabular}{|c|c|c|c|c|c|c|c|c|c|}
\hline \multirow{2}{*}{ Strain } & & \multicolumn{8}{|c|}{ Starting $\mathrm{pH}$ of the medium } \\
\hline & & pH 3 & pH 4 & pH 5 & pH 6 & pH 7 & pH 8 & $\mathrm{pH} 9$ & $\mathrm{pH} 10$ \\
\hline Rhodotorula mucilaginosa & $\mathrm{K} 12$ & 4.875 & 6.873 & 7.989 & 8.171 & 8.129 & 8.440 & 9.189 & 9.597 \\
\hline Rodosporidium fluviale & N7 & 3.330 & 7.167 & 7.504 & 7.286 & 7.294 & 7.798 & 8.535 & 9.781 \\
\hline Cryptococcus liquefaciens & $\mathrm{K} 2$ & 4.842 & 6.858 & 7.650 & 8.084 & 8.543 & 8.825 & 9.513 & 10.242 \\
\hline Candida sp. & S1 & 3.178 & 5.046 & 7.484 & 7.920 & 7.879 & 8.410 & 9.096 & 9.540 \\
\hline Candida quercuum & $\mathrm{S} 3$ & 2.980 & 3.756 & 4.826 & 7.655 & 8.813 & 8.813 & 9.368 & 10.092 \\
\hline Candida intermedia & $\mathrm{S} 4$ & 2.865 & 4.188 & 7.112 & 7.658 & 8.047 & 8.649 & 9.434 & 10.019 \\
\hline Scheffersomyces spartinae & N8 & 3.703 & 6.648 & 7.377 & 7.532 & 7.600 & 8.209 & 9.079 & 9.628 \\
\hline Wickerhamomyces anomalus & N10 & 2.929 & 4.049 & 7.059 & 7.536 & 7.925 & 8.500 & 9.321 & 9.907 \\
\hline Cyberlindnera saturnus & N11 & 4.272 & 7.433 & 7.950 & 8.199 & 8.248 & 8.580 & 9.328 & 9.830 \\
\hline
\end{tabular}

Each value is a final $\mathrm{pH}$ of the medium.

cellulosic biomass, acids or alkalis are used for the hydrolysis of the materials, and thus the direct fermentation of hydrolysates containing oligosaccharides by yeasts without neutralizing themselves is desirable, and the concentration of the biomass should be higher from an economical point of view. However, as highly concentrated hydrolysates contain high levels of oligosaccharides and rich salts and since their $\mathrm{pH}$ values are acid or alkali, the yeasts should have high fermentative activities under osmotic pressure stress, salt stress, and $\mathrm{pH}$ stress.

We previously surveyed yeasts from the coast of Tokyo Bay to isolate highly fermentative yeasts under concentrated substrates, and most of the superior yeast strains were Saccharomyces cerevisiae [16] [17] [18] [19]. We later isolated Citeromyces matritensis M37 from Tokyo Bay as a high salt-tolerant yeast that produces ethanol, and we observed its effective fermentation from salted algae [20].

The remaining problem is the application of $\mathrm{pH}$-tolerant yeasts. We have surveyed $\mathrm{pH}$-tolerant yeast strains in several types of environment. We isolated acid-tolerant yeasts from acidic or neutral streams in Japan; their classification is shown in Figure 10 [1] [2] [3]. Gadanho et al. described the yeast diversity in the extreme acidic environments of the Iberian Pyrite Belt, and they tested the yeast community in terms of high, intermediate, and low environmental stress. They identified Cryptococcus sp. under high stress ( $\mathrm{pH} 1.8$ and a high concentration of heavy metals), Rhodosporidium toruloides, Cryptococcus sp., Rhodotorula sp., and Candia fuviatilis under intermediate stress (pH 2.5 - 3.0 and intermediate concentrations of heavy metals), and Williopsis californica, Cryptococcus sp., Rhodotorula sp., Rhodotorula bogoriensis, Cryptococcus albidosimilis, and Bullera unica under low stress ( $\mathrm{pH} 2.5$ - 3.0 and low concentrations of heavy metals) [21]. Thus, there seemed to be strong similarity regarding the types of yeast species in the acidic environments between Japan and Portugal. In the present study we obtained alkali-tolerant yeasts with $\mathrm{pH}$ tolerance that was very similar to that of acid-tolerant yeasts. Acid-tolerant yeasts and alkali-tolerant 
Table 5. The yeasts used for construction of the polygenetic tree in Figure 10.

\begin{tabular}{|c|c|}
\hline \multicolumn{2}{|l|}{ Strain } \\
\hline Meyerozyma guilliermondii & mr-w1 \\
\hline Aureobasidium pullulans & $\mathrm{r}-\mathrm{w} 2,3,4, \mathrm{fi}-\mathrm{m} 10$ \\
\hline Rhodotorula sp./Leucospridium golubevii & si-w12, 13 \\
\hline Filiobasidium magunum & mi-w16 \\
\hline Hannaella pagnoccae & mi-w17 \\
\hline Cryprococcus sp. & ko-w20 \\
\hline Cryptococcus flavescens & $\mathrm{m}-\mathrm{m} 25, \mathrm{n}-\mathrm{w} 28$ \\
\hline Rhodotrula sp. & n-w29 \\
\hline Bullera alba & ks-w31 \\
\hline Pseudozyma antarctica & sm-w37 \\
\hline Hannaella coprosmae & sm-w38 \\
\hline Pseudozyma antarctica & sm-w39 \\
\hline Microbotryozyma collariae & sm-w40 \\
\hline Auriculibullersp. & om-w41 \\
\hline Cryptococcus sp. & om-w42 \\
\hline Papiliotrema flavescens & om-w44 \\
\hline Candida sp. & m-w46 \\
\hline Candida sp. & S1 \\
\hline Candida quercuum & S3 \\
\hline Candida intermedia & S4 \\
\hline Cryptococcus liquefaciens & K2 \\
\hline Rhodotorula mucilaginosa & K12 \\
\hline Rhodosporidium fluviale & N7 \\
\hline Scheffersomyces spartinae & N8 \\
\hline Wickerhamomyces anomalus & N10 \\
\hline Cyberlindnera saturnus & N11 \\
\hline
\end{tabular}

yeasts were also found to be situated in the same classification position.

\section{Conclusions}

We surveyed alkali-tolerant yeasts from natural aquatic environments at $\mathrm{pH} 7.6$ 9.4 in the Kanto region of Japan and isolated 35 strains of yeast that grew in medium at $\mathrm{pH}$ 9.0. These strains were identified as members of seven genera and nine species: 25 strains (N1, N2, N3, N4, N5, N6, N9, K1, K3, K4, K5, K6, K7, K8, K9, K10, K11, K12, K13, K14, K15, K16, K17, K18, and K19) were Rhodotorula mucilaginosa; one strain (N7) was Rhodosporidium fluviale, one strain (N8) was Scheffersomyces spartinae; two strains (N10 and N13) were Wicherhamonyces anomalus, one strain (N11) was Cyberlindnera saturnus, one strain (S1) was Candida sp.; two strains (S2 and S4) were Candida intermedia; one strain (S3) was Candida quercuum; and one strain (K2) was Cryptococcus liquefa- 
cience.

We examined the effect of $\mathrm{pH}$ on the growth of representative strains of the nine species. Strains K12 and S4 showed high growth at $\mathrm{pH} 3$ - 10. Five strains, i.e. N7, N8, N10, N11, and S3 showed high growth at pH 3 - 9. Strains K2 and S1 showed high growth at $\mathrm{pH} 4-8$. All nine strains had neutralizing activities from acidic media at $\mathrm{pH} 3-5$ to $\mathrm{pH} 6$ - 8. The alkali-tolerant yeasts and acid-tolerant yeasts [1] [2] [3] were found to be similar species, to grow well in a broad $\mathrm{pH}$ range, and to have neutralizing activities from acid media to $\mathrm{pH} 6-8$. We constructed the phylogenetic trees of the acid-tolerant strains and the alkali-tolerant strains, and all of the strains were situated in the same classification position. Similar yeast species with a broad range of $\mathrm{pH}$ tolerance were found to be living in natural aquatic environments at $\mathrm{pH}$ values from alkali to acid. Most importantly, Candida intermedia S4 showed high growth at $\mathrm{pH}$ values from 3.0 to 10.0, had fermentative activity, and seems to be a candidate for bioethanol production from cellulosic biomass.

\section{Acknowledgements}

This work was supported in part by a Japan Society for the Promotion of Science KAKENHI Grant-in-Aid for Scientific Research (No. 16K07868).

\section{Conflicts of Interest}

The authors declare no conflicts of interest regarding the publication of this paper.

\section{References}

[1] Okai, M., Suwa, C., Nagaoka, S., Obara, N., Mitsuya, D., Kurihara, A., Ishida, M. and Urano, N. (2017) Neutralization of Acidic Drainage by Cryptococcus sp. T1 Immobilized in Alginate Beads. Bioscience, Biotechnology and Biochemistry, 81, 2216-2224. https://doi.org/10.1080/09168451.2017.1373586

[2] Mitsuya, D., Hayashi, T., Wang, Y., Tanaka, M., Okai, M., Ishida, M. and Urano, N. (2017) Isolation of Aquatic Yeasts with the Ability to Neutralize Acidic Media, from an Extremely Acidic River near Japan's Kusatsu-Shirane Volcano. Journal of Bioscience and Bioengineering, 124, 43-46. https://doi.org/10.1016/j.jbiosc.2017.02.005

[3] Nagaoka, S., Kobayashi, T., Kajiwara, Y., Okai, M., Ishida, M. and Urano, N. (2017) Characterization of Yeasts Capable of Neutralizing Acidic Media from Natural Neutral Environments. Advances in Microbiology, 7, 887-897. https://doi.org/10.4236/aim.2017.712068

[4] Tanaka, K., Ishii, Y., Ogawa, J. and Shima, J. (2012) Enhancement of Acetic Acid Tolerance in Saccharomyces cerevisiae by Overexpression of the HAA1 Gene, Encoding a Transcriptional Activator. Applied and Environmental Microbiology, 78, 8161-8163. https://doi.org/10.1128/AEM.02356-12

[5] Saithong, P., Nakamura, T. and Shima, J. (2009) Prevention of Bacterial Contamination Using Acetate-Tolerant Schizosaccharomyces pombe during Bioethanol Production from Molasses. Journal of Bioscience and Bioengineering, 108, 216-219. https://doi.org/10.1016/j.jbiosc.2009.03.022

[6] Watanabe, I., Nakamura, T. and Shima, J (2008) A Strategy to Prevent the Occur- 
rence of Lactobaccillus Strains Using Lactate-Tolerant Yeast Candida glabrata in Bioethanol Production. Journal of Industrial Microbiology and Biotechnology, 35, 1117-1122. https://doi.org/10.1007/s10295-008-0390-1

[7] Serra-Cardona, A., Canadell, D. and Arino, J. (2015) Coordinate Responses to Alkaline $\mathrm{pH}$ Stress in Budding Yeast. Microbial Cell, 2, 182-196.

https://doi.org/10.15698/mic2015.06.205

[8] Saito, M., Nagasaki, H., Ikeda, T., Saga, K., Yoshida, K. and Watanabe S. (2018) Pretreatment Conditions for Hydrolysates from Unbleached Pulp Waste for Ethanol Fermentation with Xylose-fermenting Yeast, Candida intermedia 4-6-4T2. Journal of the Japan Petroleum Institute, 61, 191-198. https://doi.org/10.1627/jpi.61.191

[9] Lisichkina, G.A., Bab'eva, L.P. and Sorokin, D.Yu. (2003) Alkalitolerant Yeasts from Natural Biotope. Microbiology, 72, 695-698.

https://doi.org/10.1023/A:1026011821021

[10] Takagi, T., Uchida, M., Matsushima, R., Kodama, H., Takeda, T., Ishida, M. and Urano, N. (2015) Comparison of Ethanol Productivity among Yeast Strains Using Three Different Seaweeds. Fisheries Science, 81, 763-770.

https://doi.org/10.1007/s12562-015-0875-6

[11] Obara, N., Okai, M., Ishida, M. and Urano, N. (2015) Bioethanol Production from Mixed Miomass (Waste of Undaria pinnafida Processing and Paper Shredding) by Fermentation with Marine-Derived Saccharomyces cerevisiae. Fisheries Science, 81, 771-776. https://doi.org/10.1007/s12562-015-0877-4

[12] Takagi, T., Adachi, S., Higashi, M., Kawaguchi, R., Uchida, M., Matsushima, R., Ishida, M. and Urano, N. (2012) Bioethanol Production from Seaweeds Using Aquatic Yeasts. The 9th Asia-Pacific Marine Biotechnology Conference Oral Section, Kochi, 14 July 2012.

[13] Takagi, T., Uchida, M., Matsushima, R., Ishida, M. and Urano, N. (2012) Efficient Bioethanol Production from Water Hyacinth Eichhornia crassipes by Both Preparation of the Saccharified Solution and Selection of Fermenting Yeasts. Fisheries Science, 78, 905-910. https://doi.org/10.1007/s12562-012-0516-2

[14] Ogawa, G., Ishida, M., Usui, Y. and Urano, N. (2008) Ethanol Production from the Water Hyacinth Eichhornia crassipes by Yeasts Isolated from Various Hydrospheres. African Journal of Microbiology Research, 2, 110-113.

[15] Obara, N., Ishida, M., Hamada-Sato, N. and Urano, N. (2012) Efficient Bioethanol Production from Paper Shredder Scrap by a Marine Derived Saccharomyces cerevisiae C-19. Studies in Science and Technology, 1, 127-132.

[16] Obara, N., Oki, N., Okai, M., Ishida, M. and Urano, N. (2015) Development of a Simple Isolation Method for Yeast Saccharomyces cerevisiae with High Fermentative Activity from Coastal Waters. Studies in Science and Technology, 4, 71-76.

[17] Ogawa, G., Ishida, M., Shimotori, K. and Urano, N. (2008) Isolation and Characterization of Saccharomyces cerevisiae from Hydrospheres. Annals of Microbiology, 58, 262-264. https://doi.org/10.1007/BF03175326

[18] Urano, N., Shirao, A., Okai, M. and Ishida, M. (2017) High Ethanol Production by Marine-Derived Saccharomyces cerevisiae under Stress Pressures. Advances in Microbiology, 7, 349-357. https://doi.org/10.4236/aim.2017.75029

[19] Mitsuya, D., Okai, M., Kawaguchi, R., Ishida, M. and Urano, N. (2016) Cascade Bioethanol Productions from Glucose and Mannitol in Saccharified Kombu Laminariaceae by Anaerobic and Aerobic Fermentations with Two Kinds of Yeast. Studies in Science and Technology, 5, 59-65. 
[20] Okai, M., Bestuno, A., Shirao, A., Obara, N., Suzuki, K., Takei, T., Takashio, M., Ishida, M. and Urano, N. (2017) Citromyces matritensis Is a Salt Tolerant Yeast That Produces Ethanol from Salted Algae. Canadian Journal of Microbiology, 63, 20-26. https://doi.org/10.1139/cjm-2016-0259

[21] Gadanho, M., Libkind, D. and Sampaio, J.P. (2006) Yeast Diversity in the Extreme Acidic Environments of the Iberian Pyrite Belt. Microbial Ecology, 52, 552-563.

https://doi.org/10.1007/s00248-006-9027-y 